\title{
A SECOND ORDER DIFFERENTIAL EQUATION OF T. OTSUKI
}

\author{
W. R. UTZ
}

\begin{abstract}
It is shown that an ordinary differential equation arising in the study of minimal hypersurfaces in certain Riemannian manifolds and for which one family of periodic solutions is known has, in fact, a second family of periodic solutions.
\end{abstract}

1. T. Otsuki has shown [2] that the equation

$$
n h\left(1-h^{2}\right) \frac{d^{2} h}{d t^{2}}+\left(\frac{d h}{d t}\right)^{2}+\left(1-h^{2}\right)\left(n h^{2}-1\right)=0
$$

plays a vital role in the study of geodesics on certain Riemannian manifolds. Solutions of (1) for which

$$
h^{2}+\left(h^{\prime}\right)^{2}<1, \quad n=2,3,4, \ldots,
$$

are periodic and of particular interest geometrically. These solutions have been studied by Otsuki [3], [4], S. Furuya [1] and M. Urabe [5].

Heretofore equation (1) has only been studied for integral values of $n>2$ and $h^{2}+\left(h^{\prime}\right)^{2}<1$ since these are the conditions that provide immediate geometric applications. In this note we consider (1) for all real $n$ and without the restriction $h^{2}+\left(h^{\prime}\right)^{2}<1$. In doing so we discover another family of periodic solutions of (1).

2. If $h(t)$ satisfies (1), then so does $-h(t)$ and for this reason we confine our attention to solutions for which $h(t)>0$. Equation (1) has the constant solution $h(t) \equiv 1$ for all real $n, h(t) \equiv 1 / \sqrt{n}$ for all real $n>0$ and, for all $n$, the periodic solutions $h(t)=\sin (t+\omega), h(t)=\cos (t+\omega)$.

It is convenient to set $h^{\prime}(t)=v$ whereupon (1) impliies

$$
v^{2}=1-h^{2}-C\left(1 / h^{2}-1\right)^{1 / n}
$$

We will consider the family of lines

$$
y=(1-x) / C
$$

as they cut the curve

$$
y=(1 / x-1)^{1 / n}
$$

since these intersections reveal the zeros of $v(t)$.

Presented to the Society, January 27, 1977; received by the editors April 2, 1976.

AMS (MOS) subject classifications (1970). Primary 35B 10. 
3. To completely treat the behavior of the solutions of (1) it is appropriate to consider the cases $n>1, n=1,0<n<1, n=0$ and $n<0$. The case $n=0$ is trivial. In the other cases there are two subcases, viz., $0<h(t)<1$ and $h(t)>1$. Phase-plane portraits in each of these cases are not difficult to obtain provided, in case $h(t)>1, n$ is such that

$$
\left(1 / h^{2}-1\right)^{1 / n}
$$

is real. For this reason, when $h(t)>1$ we treat the cases of rational $n$ 's with odd numerators.

Rather than give a definitive analysis of all cases we concentrate on the case $0<n<1$ since it is for such $n$ that one finds the other families of bona fide periodic solutions (in other cases families of ovals occur but with a singularity, i.e., a common point of tangency for the entire family).

4. In this section we assume that $0<n<1$. (a). If $0<h<1$ and $n$ is any real number in the assigned range, then the curve (4) is asymptotic to the $y$-axis and falls monotonically with positive concavity to meet the $x$-axis at $x=1$. Thus, (3) and (4) intersect at two places, $x=a(C)<1$ and $x=1$. Between these values (of $h$ ) $v^{2}>0$ and thus the strip $0<h<1$ of the $(h, v)$-plane is filled with simple closed curves given by (2). All curves of the family contain the point $(h=1, v=0)$.

(b). Now, consider $0<n<1, h>1, n=p / q$ where $p$ and $q$ are relatively prime integers. Clearly, the graph of (2) is not real if $p$ is even and so we hereafter take $p$ as an odd integer.

If $q$ is odd, the curve (4) cuts the $x$-axis at $x=1$ where it is tangent to the axis. The concavity of (4) changes from up to down at $x=1$ and so immediately to the right of $x=1,(4)$ is concave down. There is an inflection point at $x=(p+q) / 2 p>1$ past which (4) is concave up and then asymptotic to $y=-1$.

The line (3) intersects $(4)$ at $(1,0)$ for all values of $C$. For

$$
0<C<\frac{q}{p}\left(\frac{p}{q}-1\right)^{1-q / p}
$$

the line (3) lies below (4) for all $x>1$, it is tangent to (4) for

$$
C_{1}=\frac{q}{p}\left(\frac{q}{p}-1\right)^{1-q / p} \text {. }
$$

The point of tangency is

$$
x=\frac{q}{p}, \quad y=\left(\frac{p}{q}-1\right)^{q / p} .
$$

For each $C>C_{1}$ (3) cuts (4) in two points $x=a(C)>1, x=b(C)>a(C)$ and for $a<x<b$ the line (3) is above the curve (4); hence $v^{2}>0$ and an oval results in the $(h, v)$-plane corresponding to a periodic solution of (1).

By simply taking an arbitrary $h>1$ and any $h^{\prime}=v$ one discovers that 
these ovals fill the region $h^{\prime}>1$. It is clear that $a(C) \rightarrow 1$ and $b(C) \rightarrow \infty$ as $C \rightarrow \infty$ and that the ovals have as "center" the $x$-coordinate corresponding to the $x$-coordinate of the point of tangency of the member of (3) tangent to (4). That is, the point has $x$-coordinate $a\left(C_{1}\right)=b\left(C_{1}\right)=q / p$. Thus, the "center" in the $(h, v)$-plane is $(\sqrt{q / p}, 0)$, that is, $(1 / \sqrt{n}, 0)$. The solutions just revealed are genuine periodic solutions (i.e., they are mutually disjoint ovals).

In case $p$ is odd and $q$ is even, the same phase-portrait results. In this case $C<0$, (4) is tangent to the $x$-axis at $(1,0)$ and rises monotonically to be asymptotic to $y=1$. Ovals corresponding to periodic solutions fill $h>1$ and surround the point $(x=q / p, y=0)$. That is, $(h=\sqrt{q / p}, v=0)$ is, again, the center of the ovals.

We now summarize the principal observation of this note.

THEOREM. If $n=p / q$ where $p$ and $q$ are integers, $p$ odd, then given initial conditions $h(0)>1, h^{\prime}(0)$ arbitrary, there corresponds a periodic solution of (1) satisfying these intitial conditions.

5. Having seen the case $0<n<1$ it is evident as to how one may proceed to treat all cases and these are left to the reader. It may be worth recording that (in addition to the positive integral cases considered by Otsuki, et al.) for all real $n>1$ and $h^{2}+\left(h^{\prime}\right)^{2}<1$ it follows that all solutions are periodic. Indeed, the arguments of Otsuki may be applied in this case.

\section{REFERENCES}

1. S. Furuya, On periods of periodic solutions of certain nonlinear differential equations (JapanUnited States Seminar on Ordinary Differential and Functional Equations), Lecture Notes in Math., vol. 243, Springer-Verlag, Berlin and New York, 1971, pp. 320-323.

2. T. Otsuki, Minimal hypersurfaces in a Riemannian manifold of constant curvature, Amer. J. Math. 92 (1970), 145-173. MR 41 \#9157.

3. $ـ$ On integral inequalities related with a certain nonlinear differential equation, Proc. Japan Acad. 48 (1972), 7-12. MR 46 \#7635.

4. _ On a bound for periods of solutions of certain nonlinear differential equations. I, J. Math. Soc. Japan 26 (1974), 206-233.

5. M. Urabe, Computations of periods of a certain nonlinear autonomous oscillation. Study of algorithms of numerical computations, Sûrikaiseki Kenkyûsho Kôkyû-roku 148 (1972), 111-129. (Japanese)

Department of Mathematics, University of Missouri, Columbia, Missouri 65201 